\title{
Rate of disease progression: a prognostic biomarker in ALS
}

\author{
Julie Labra, ${ }^{1}$ Parvathi Menon, ${ }^{2}$ Karen Byth, ${ }^{3,4}$ Shea Morrison, ${ }^{1}$ Steve Vucic ${ }^{2}$
}

\begin{abstract}
${ }^{1}$ St Joseph's Hospital, Sydney, Australia

${ }^{2}$ Western Clinical School, University of Sydney, Sydney, Australia

${ }^{3}$ Westmead Hospital, Research and Education Network, Sydney, Australia

${ }^{4}$ NHMRC Clinical Trials Centre, University of Sydney, Sydney, Australia
\end{abstract}

\section{Correspondence to}

Professor Steve Vucic, Department of Neurology,

Westmead Hospital, Cnr

Hawkesbury and Darcy Roads,

Westmead, NSW 2145,

Australia;

s.vucic@neura.edu.au

Received 7 April 2015 Accepted 22 June 2015 Published Online First 7 July 2015
CrossMark

To cite: Labra J, Menon $P$, Byth K, et al. I Neurol Neurosurg Psychiatry 2016;87:628-632.
ABSTRACT

Objective To assess the utility of rate of disease progression ( $\triangle \mathrm{FS}$ ) as a prognostic biomarker in amyotrophic laterals sclerosis (ALS).

Methods A total of 203 patients with ALS were prospectively recruited over a 10-year period. At initial visit, the following variables were collected: demographic details, symptom duration, site of onset, phenotype, riluzole use and Amyotrophic Lateral Sclerosis Functional Rating Scale-Revised (ALSFRS-R) scores. The $\triangle$ FS score at initial visit was calculated by dividing the ALSFRS-R total score by symptom duration (months). The primary end point was survival. Kaplan-Meier survival curves were used to illustrate the distribution of survival from a specified point, while multiple Cox proportional hazards modelling with backward stepwise variable selection was used to identify the independent predictors of survival at initial visit.

Results The $\Delta F S$ score at initial visit was a significant predictor of survival in ALS $(p<0.001)$, and remained significant when adjusted for age and site of onset $(p<0.001) .3$ prognostic subgroups emerged, with a $\Delta F S$ score of $<0.47$ associated with a median survival of 2.4 years, which was significantly greater when compared with an initial $\Delta \mathrm{FS}$ score of between 0.47 and 1.11 (1.6 years, $p<0.05$ ) and a score $>1.11(0.7$ years, $\mathrm{p}<0.001$ ). Importantly, multiple Cox proportional hazards modelling identified $\Delta \mathrm{FS}$ as a highly significant independent predictor of survival in ALS $(p<0.001)$ along with site of disease onset $(p<0.01)$.

Conclusions Rate of disease progression appears to be a simple and sensitive clinical prognostic biomarker in ALS that could be potentially utilised in clinical practice and future therapeutic trials.

\section{INTRODUCTION}

The development of clinically applicable and reliable prognostic biomarkers in amyotrophic lateral sclerosis (ALS) would be of vital importance for guiding patient management and recruitment into clinical treatment trials. ${ }^{1}$ In a clinical setting, the availability of useful prognostic biomarkers would enable implementation of an appropriate care plan. ${ }^{2}$ From a research perspective, the identification of sensitive and clinically applicable biomarkers would enable equal stratification of patients into treatment groups (active vs placebo) based on the rate of disease progression, and provide an opportunity to promptly evaluate drug effectiveness.

A number of demographic biomarkers including older age, bulbar-onset disease, respiratory dysfunction and nutritional status have been correlated with adverse prognosis in ALS. ${ }^{2-5}$ The limitation in utilising these clinical biomarkers pertains to heterogeneity of the ALS phenotype, particularly in determining the differences in disease progression rates within patients. ${ }^{6}$ Of further relevance, blood, cerebrospinal fluid, neurophysiological and neuroradiological biomarkers have also been reported, although the reliability and clinical applicability of such biomarkers in ALS remains limited. ${ }^{1}$

The Amyotrophic Lateral Sclerosis Functional Rating Scale-Revised (ALSFRS-R) has been reported as a prognostic biomarker predictor of survival in ALS. ${ }^{7}$ A potential limitation of total ALSFRS-R score is the failure to incorporate symptom duration, an independent predictor of survival. ${ }^{9}{ }^{10}$ Consequently, the rate of disease progression $(\triangle \mathrm{FS})$ score was developed which expressed the ALSFRS-R as a function of the disease duration. ${ }^{11}$ A study in a Japanese ALS cohort suggested that the $\Delta \mathrm{FS}$ at initial assessment was an independent and reliable biomarker of disease progression in individual patients when compared with the ALSFRS-R, ${ }^{11}$ a finding confirmed in a subsequent study. ${ }^{12}$ The prognostic utility of $\Delta \mathrm{FS}$ requires further validation, particularly the development of statistically derived prognostic cut-off values, prior to being accepted as a useful prognostic biomarker. Consequently, the present study assessed the utility of $\Delta \mathrm{FS}$ as a prognostic clinical biomarker in a large Australian ALS cohort, with the aim of developing reliable prognostic groups.

\section{METHODS \\ Patients}

The ALS cohort was prospectively recruited between 1 June 2004 and 31 July 2014, and registered on the St Joseph's Hospital ALS database, a contributor to the Australian motor neuron disease registry (AMNDR), the largest clinical ALS database in Australia. ${ }^{13}$ The cohort in the ALS database comprised 203 patients with ALS. All patients were clinically assessed in the multidisciplinary ALS clinic every 3-6 months, depending on clinical need, and were followed up until death or census date (31 July 2014). The primary end point was survival from initial visit, calculated by time to death or disease duration at census date. This study was approved by the Sydney West Area Health Service and the St Vincent's Hospital Sydney Human Research Ethics Committees.

Data was collected for the following variables: gender, age at initial visit (years), symptom onset date (obtained from patient or family reports) and disease duration at initial visit in clinic, diagnosis 
date, phenotype, ALSFRS-R total score, ${ }^{7}$ percutaneous endoscopic gastrostomy (PEG) tube insertion and institution of noninvasive ventilation. The rate of disease progression $(\Delta \mathrm{FS})$ was calculated at the initial visit using the following formula: ${ }^{11}$

$$
\frac{48-(\text { Total ALSFRS }-\mathrm{R} \text { at initial visit })}{\text { Symptom duration (months) }}
$$

\section{Statistical analysis}

All statistical analyses were performed using the SPSS Statistics package (V.21), and the analyses were exploratory. Two-tailed tests with a significance level of $5 \%$ were used throughout. Continuous variables were often skewed and are summarised using the median and lower quartile (LQ) to upper quartile (UQ). The $\chi^{2}$ or exact permutation tests were used as appropriate to test for association between categorical variables. Kruskal-Wallis non-parametric analysis of variance was used to assess differences between means. Kaplan-Meier survival curves were used to illustrate the distribution of survival from a specified point, and log rank tests were used to test for differences between groups in the survival from initial visit. To facilitate these analyses, potential risk factors with continuous distributions were split into categories as follows: age $(<70, \geq 70$ years), $\Delta \mathrm{FS}$ tertiles $(<0.47,0.47-1.11,>1.11)$, ALSFRS-R quartiles $(\leq 33,34-39,40-43, \geq 44)$, ALSFRS-R respiratory subscore tertiles $(<10,10-11,12)$ and symptom duration quartiles $(<0.7$, $0.7-1,1.1-2,>2$ years). Univariable analysis was initially performed to assess which covariates were associated with survival. All potential risk factors demonstrating a univariable association with survival from initial visit were considered as candidate variables for inclusion in a multiple Cox proportional hazards model with backward stepwise variable selection, in order to identify the independent predictors of survival from initial visit. HR with 95\% CIs were used to quantify the degree of association between a risk factor and survival from initial visit in clinic.

\section{RESULTS}

The study was undertaken on 203 patients with ALS (men 111, women 92, median age 63 years, age range $24-89$ years) diagnosed in accordance with the revised El Escorial criteria. ${ }^{14}$ Seventy-two per cent of patients were classified in the El Escorial definite/probable diagnostic categories, while $28 \%$ were classified as possible ALS. Thirty-six patients were excluded from the study due to an incomplete data set, and these patients were not clinically different from the ALS cohort recruited into the study. Three patients were excluded due to the fact that a non-ALS neuromuscular disorder (two with Kennedy's disease and one with autoimmune motor neuropathy) was established during follow-up. Consequently, the cohort for this study comprised 164 registered patients with ALS (93 men, 71 women, median age 65 years, range $26-89$ years) and their clinical features are summarised in table 1.

The median age of patients at initial visit was 64.7 years (58-73 years), with the median disease duration from symptom onset to assessment in clinic being 12 months (7.2, 24 months). In order to ensure that the timing of symptom onset was accurate, information was also sought from patients' relatives. Limb-onset disease was evident in 58\% of patients with ALS, while bulbaronset disease was reported in $42 \%$ patients. At initial assessment, the median ALSFRS-R score was 39 (33-43), while the median $\Delta \mathrm{FS}$ score was $0.67(0.33-1.47)$. Seventy-seven per cent of patients were receiving riluzole at or during the follow-up period. During the follow-up period, $49 \%(\mathrm{~N}=81)$ of patients underwent a PEG insertion at a median time of 17.5 months (12-28 months) after symptom onset, while $21 \%(\mathrm{~N}=34)$ of patients were started on non-invasive ventilation at a median time of 20.0 months (1236 months) after symptom onset. None of the patients had tracheostomy-assisted ventilation.

Table 1 Clinical characteristics for patients with amyotrophic lateral sclerosis (ALS) as a group and divided into three tertiles as defined by the rate of disease progression $(\Delta \mathrm{FS})$

\begin{tabular}{|c|c|c|c|c|}
\hline Characteristic & $\begin{array}{l}\text { All } \\
n=164\end{array}$ & $\begin{array}{l}\Delta \mathrm{FS} \text { group } 1(<0.47) \\
\mathrm{n}=56\end{array}$ & $\begin{array}{l}\Delta \mathrm{FS} \text { group } 2(0.47-1.11) \\
\mathrm{n}=54\end{array}$ & $\begin{array}{l}\Delta \mathrm{FS} \text { group } 3(>1.11) \\
\mathrm{n}=54\end{array}$ \\
\hline \multicolumn{5}{|l|}{ Gender (\%) } \\
\hline Male & 57 & 55 & 65 & 50 \\
\hline Female & 43 & 45 & 35 & 50 \\
\hline \multicolumn{5}{|l|}{ Site of onset (\%) } \\
\hline Limb & 55 & 66 & 54 & 44 \\
\hline Bulbar & 44 & 33 & 46 & 54 \\
\hline Respiratory & 1 & 2 & 0 & 2 \\
\hline \multicolumn{5}{|l|}{ Phenotype (\%) } \\
\hline ALS & 84 & 74 & 83 & 96 \\
\hline ALS-FTD & 4 & 0 & 12 & 0 \\
\hline Flail-arm variant & 4 & 10 & 0 & 2 \\
\hline PBP & 4 & 4 & 6 & 2 \\
\hline PLS & 3 & 8 & 0 & 0 \\
\hline PMA & 1 & 4 & 0 & 0 \\
\hline Median age at visit 1 & $64.7(58,73)$ & $63.3(58,71)$ & $64.7(59,75)$ & $69.4(58,75)$ \\
\hline Symptom duration (years) & $1.0(0.6,2)$ & $2.2(1.2,4.9)$ & $1.0(0.6,1.5)$ & $0.6(0.4,0.9)$ \\
\hline Median ALSFRS score visit 1 & $39(33,43)$ & $42(36,44)$ & $40(36,43)$ & $34(26,38)$ \\
\hline Median $\Delta \mathrm{FS}$ score at visit 1 & $0.67(0.33,1.47)$ & $0.24(0.13,0.34)$ & $0.67(0.60,0.94)$ & $2.69(1.47,4)$ \\
\hline
\end{tabular}

The median Amyotrophic Laterals Sclerosis Rating Scale-Revised (ALSFRS-R) was significantly lower in the highest tertile $\Delta$ FS group. Atypical ALS phenotypes such as primary bulbar palsy (PBP), primary lateral sclerosis (PLS) and progressive muscular atrophy (PMA) were infrequent, but more common in the lowest $\triangle \mathrm{FS}$ group. All data is expressed as median (IQR). FTD, frontotemporal dementia. 
In total, $77 \%$ of patients with ALS had died by the census date (31 July 2014). Utilising Kaplan-Meier plots, the median survival from symptom onset was estimated to be 2.7 years (95\% CI 2.3 to 3.1 years), while the median survival time from diagnosis of ALS was 1.7 years (95\% CI 1.5 to 2.0 years), and that from initial visit to the clinic 1.4 years $(95 \% \mathrm{CI} 1.2$ to 1.6 years). In addition, the percentage of patients surviving at 1 , 2,3 and 5 years after initial assessment in the clinic was 59 $\pm 3.9 \%, 33 \pm 3.8 \%, 23 \pm 3.6 \%$, and $21 \pm 3.5 \%$, respectively, and is in keeping with previous studies. 31015

\section{Prognostic biomarkers in ALS}

In order to determine whether $\Delta \mathrm{FS}$ was a prognostic biomarker of ALS at initial assessment, a univariable analysis was performed. The rate of disease progression at initial assessment was a significant predictor of survival in patients with ALS when assessed at initial visit $(p<0.001$, table 2$)$. The association remained highly significant when adjusted for age and site of onset $(p<0.001)$. Specifically, a $\Delta F S$ score of $<0.47$ at initial

Table 2 Univariable survival analysis disclosed that a higher rate of disease progression ( $\Delta \mathrm{FS})$, along with lower Amyotrophic Laterals Sclerosis Rating Scale-Revised (ALSFRS-R) score, older age of onset ( $\geq 70$ years), bulbar onset disease and shorter disease duration were predictors of survival

\begin{tabular}{|c|c|c|c|c|c|}
\hline $\begin{array}{l}\text { Predictor } \\
\text { variable }\end{array}$ & Number & $\begin{array}{l}\text { Median survival } \\
\text { time from initial } \\
\text { visit (years) }\end{array}$ & HR & $95 \% \mathrm{Cl}$ & $\mathrm{p}$ Value \\
\hline \multicolumn{6}{|l|}{ Gender } \\
\hline Male & 93 & 1.4 & 1 & & \\
\hline Female & 71 & 1.4 & 0.92 & 0.64 to 1.30 & 0.626 \\
\hline \multicolumn{6}{|l|}{ Age } \\
\hline$<70$ & 104 & 1.6 & 1 & & \\
\hline$\geq 70$ & 60 & 1 & 1.77 & 1.23 to 2.55 & 0.002 \\
\hline \multicolumn{6}{|l|}{ Site of onset } \\
\hline Limb & 95 & 1.6 & 1 & & \\
\hline Bulbar & 69 & 0.9 & 1.76 & 1.23 to 2.51 & 0.002 \\
\hline \multicolumn{6}{|c|}{ Symptom duration (years) } \\
\hline 0.7 & 41 & 1.3 & 2.52 & 1.45 to 4.39 & $<0.001$ \\
\hline $0.7-1$ & 42 & 1.4 & 2.74 & 1.61 to 4.68 & $<0.001$ \\
\hline $1.1-2$ & 40 & 1.2 & 2.81 & 1.62 to 4.89 & $<0.001$ \\
\hline$>2$ & 41 & 5 & 1 & & $<0.001$ \\
\hline \multicolumn{6}{|l|}{ Riluzole } \\
\hline Yes & 127 & 1.5 & 0.7 & 0.46 to 1.05 & 0.087 \\
\hline No & 37 & 0.7 & 1 & & \\
\hline \multicolumn{6}{|l|}{ PEG tube } \\
\hline Yes & 81 & 1.4 & 1.36 & 0.96 to 1.94 & 0.09 \\
\hline No & 83 & 1.5 & 1 & & \\
\hline \multicolumn{6}{|l|}{ NIV } \\
\hline Yes & 34 & 1.3 & 1.24 & 0.83 to 1.85 & 0.304 \\
\hline No & 130 & 1.4 & 1 & & \\
\hline \multicolumn{6}{|l|}{$\Delta \mathrm{FS}$ tertiles } \\
\hline$<0.47$ & 56 & 2.4 & 1 & & \\
\hline $0.47-1.11$ & 54 & 1.6 & 1.77 & 1.12 to 2.82 & 0.015 \\
\hline$>1.11$ & 54 & 0.7 & 3.74 & 2.40 to 5.82 & $<0.001$ \\
\hline \multicolumn{6}{|c|}{ ALSFRS-R quartiles } \\
\hline$\leq 33$ & 48 & 0.6 & 2.37 & 1.40 to 4.04 & 0.002 \\
\hline $34-39$ & 42 & 1.5 & 1.65 & 0.94 to 2.92 & 0.084 \\
\hline $40-43$ & 42 & 1.5 & 1.72 & 0.99 to 3.01 & 0.056 \\
\hline$\geq 44$ & 32 & 2.6 & 1 & & \\
\hline
\end{tabular}

The presence of a percutaneous endoscopic gastrostomy (PEG) tube and non-invasive ventilation did not significantly impact on survival in the present cohort. assessment was associated with a median survival of 2.4 years, which was significantly greater when compared with an initial $\Delta \mathrm{FS}$ score of between 0.47 and 1.11 (1.6 years, HR $1.77,95 \%$ CI 1.12 to $2.82, \mathrm{p}<0.05)$ and a score $>1.11(0.7$ years, HR 3.74 , CI 2.40 to $5.82, \mathrm{p}<0.001$, figure 1 , table 2 ).

Subgroup analysis of the three $\Delta F S$ groups disclosed a greater proportion of patients with bulbar onset disease in the highest tertile group $(\Delta \mathrm{FS}>1.11)$ as well as a greater frequency of the classical ALS phenotype (table 1$)$. In addition, patients in the highest tertile groups were significantly older ( $\geq 70$ years), and exhibited a significantly shorter symptom duration and time to definite/probable ALS diagnosis (table 1). Of further relevance, the total ALSFRS-R score was significantly smaller (table 2), as were the ALSFRS subscores (figure 2). Importantly, the proportion of patients receiving riluzole in the highest tertile group was not significantly different from more favourable tertile groups (table 1).

In addition to the rate of disease progression score, older age $\geq 70$ years, shorter duration of symptoms, lower total ALSFRS R score, ALSFRS-R respiratory subscore $<10$, and bulbar onset disease at initial visit were also identified as adverse prognostic biomarkers in ALS on univariable analysis (table 2). By contrast, gender, therapy with riluzole, presence of percutaneous endoscopic gastrostomy (PEG) tube or non invasive ventilation (NIV) use were not significant predictors of survival in the present cohort (table 2).

Since several variables were associated with survival from initial visit on univariable analysis, multiple Cox proportional hazards modelling was used to explore the relationship between survival in ALS and six candidate variables: age at initial visit ( $<70$ vs $\geq 70$ years), site of onset (limb vs bulbar), symptom duration at initial visit quartiles (years), ALSFRS-R total score, ALSFRS-R respiratory subscore and $\triangle F S$ tertiles. The best-fitting Cox proportional hazards model obtained using backward stepwise variable selection, identified $\Delta F S$ tertiles at initial assessment and the site of disease onset as independent significant predictors of survival in ALS (table 3).

\section{DISCUSSION}

The findings in the present study have further validated the prognostic utility of the $\Delta \mathrm{FS}$ score at initial assessment in an

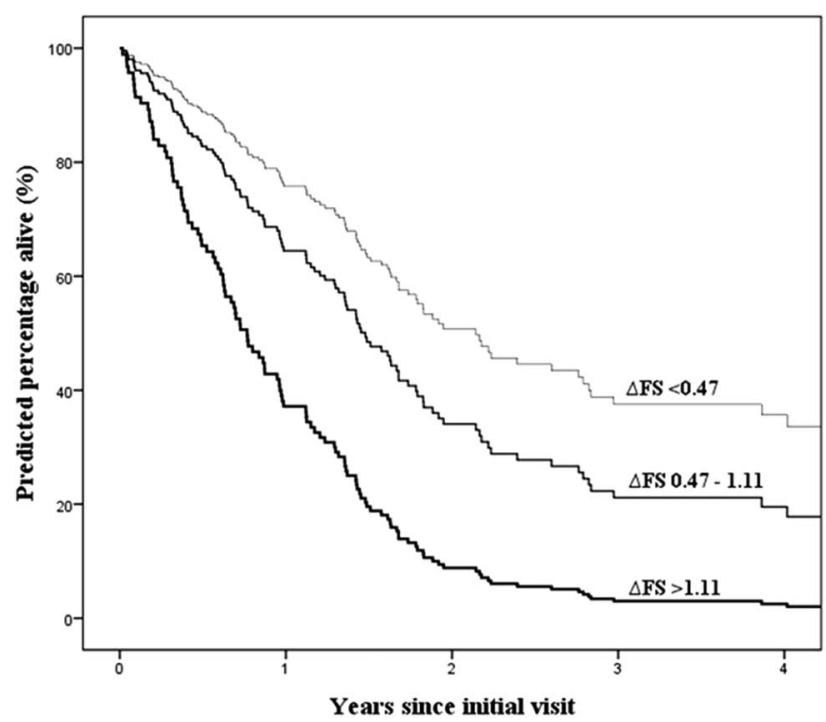

Figure 1 Kaplan-Meier survival curves indicating that a rate of disease progression $(\Delta \mathrm{FS})<1.11$ at initial assessment was associated with poorest survival. 

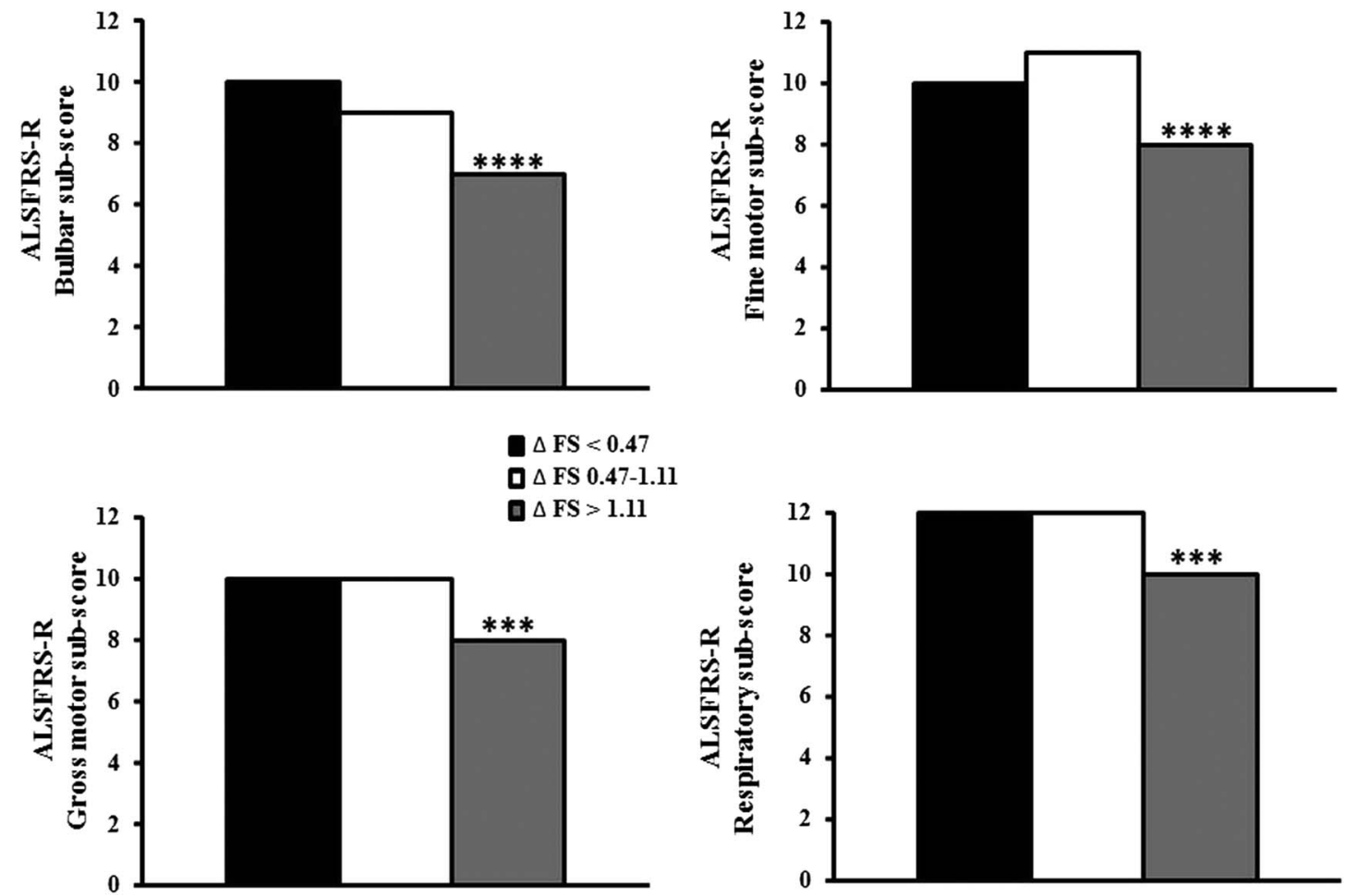

Figure 2 The median Amyotrophic Laterals Sclerosis Rating Scale-Revised (ALSFRS-R) subscores were significantly smaller in patients with the highest rate of disease progression $\left(\Delta \mathrm{FS} ;{ }^{* * *} \mathrm{p}<0.001 ;{ }^{* * * *} \mathrm{p}<0.0001\right)$.

Australian ALS cohort, establishing $\triangle \mathrm{FS}$ as an independent and robust prognostic biomarker for ALS. In addition, the three statistically derived prognostic groups emerged $(\Delta \mathrm{FS}<0.47,0.47-$ 1.11 and $>1.11$ ), not previously reported, could be potentially utilised as prognostic guides in patients management and future clinical trials. Importantly, a $\Delta \mathrm{FS}$ score of $>1.11$ resulted in survival times of only 8.4 months and a 3.7 -fold greater risk of death, when compared with patients with a $\Delta \mathrm{FS}$ score $<0.47$. In addition, older age ( $\geq 70$ years), shorter disease duration ( $\leq 2$ years), lower total ALSFRS-R score $(\leq 33)$, ALSFRS-R respiratory subscore $<10$ and bulbar-onset disease were also adverse prognostic factors on univariable analysis. Importantly, multiple Cox proportional hazard modelling identified $\Delta \mathrm{FS}$ at initial clinic visit and bulbar-onset disease as independent predictors of survival in ALS. These results suggest a potential utility of $\Delta \mathrm{FS}$ as a prognostic biomarker in ALS, as it robustly discriminates between three prognostic subgroups.

Table 3 Best fitting Cox proportional hazards model showing independent predictors of survival at initial visit

\begin{tabular}{|c|c|c|c|}
\hline Predictor variable & HR & $95 \% \mathrm{Cl}$ & p Value \\
\hline \multicolumn{4}{|l|}{ Age(year) } \\
\hline$<70$ & 1 & & \\
\hline$\geq 70$ & 1.44 & 0.99 to 2.11 & 0.060 \\
\hline \multicolumn{4}{|l|}{ Site of onset } \\
\hline Limb & 1 & & \\
\hline Bulbar & 1.62 & 1.13 to 2.33 & $<0.05$ \\
\hline \multicolumn{4}{|l|}{$\Delta \mathrm{FS}$ tertiles } \\
\hline$<0.47$ & 1 & & \\
\hline $0.47-1.11$ & 1.63 & 1.09 to 2.26 & $<0.05$ \\
\hline$>1.11$ & 3.05 & 1.93 to 4.83 & $<0.001$ \\
\hline
\end{tabular}

\section{Prognostic biomarkers in ALS}

The development of reliable and sensitive prognostic biomarkers for ALS is of vital importance in patient management and stratification into clinical trials. ${ }^{1}$ Specifically, identifying patients with aggressive disease at early stages of ALS may enable more adequate patient care planning, including timing of institution of communication devices, gastrostomy feeding and noninvasive ventilation. ${ }^{16}$ In addition, prognostic biomarkers may increase the power of clinical trials by reducing subject heterogeneity, ${ }^{17}$ and could be utilised as primary end points, assessing the pharmacodynamic effect of drugs in early stages of development. Ideally, a prognostic biomarker should reliably discriminate between ALS subgroups, and be easily accessible and practical to implement. ${ }^{1}$

The findings in the present study establish $\Delta \mathrm{FS}$ at initial clinic visit, as a sensitive prognostic biomarker in ALS. Specifically, the $\Delta \mathrm{FS}$ robustly discriminates between three different prognostic ALS subgroups, as indicated by multiple Cox proportional hazard models where $\triangle \mathrm{FS}$ was an independent predictor of survival. In addition, $\Delta \mathrm{FS}$ is simple and inexpensive to administer, requiring disease duration and the ALSFRS-R score, both of which are routinely clinically collated. Importantly, the present findings are in keeping with previous studies which have identified $\Delta \mathrm{FS}$ as a prognostic biomarker for ALS survival at initial assessment. ${ }^{11} 12$

The main limitation in utilising the $\Delta \mathrm{FS}$ as a prognostic biomarker in ALS pertains to an absence of universally accepted prognostic values. Previous studies have assessed the utility of $\Delta \mathrm{FS}$ by dividing patients into two groups about a median $\Delta \mathrm{FS}$ value, and have arbitrarily assigned three prognostic cut-off values $(<0.5 ; 0.5-1$ and $\geq 1) .{ }^{5} 9$ In addition, it has also been suggested that $\triangle \mathrm{FS}$ scores may exhibit a regional variation, with the suggestion that centres should develop unique $\Delta \mathrm{FS}$ score cut-off values, thereby potentially limiting the $\Delta \mathrm{FS}$ score as a ubiquitous prognostic biomarker in ALS. It is reassuring that the 
median $\Delta \mathrm{FS}$ score of 0.67 in the present study was similar to previous findings, ${ }^{11}$ as were the three statistically derived prognostic cut-off values $(<0.47,0.47-1.11,>1.11)$. As such, the $\Delta \mathrm{FS}$ score prognostic values appear to be similar irrespective of the origins of the ALS cohorts, thereby supporting the utility of the $\triangle \mathrm{FS}$ score as a prognostic biomarker in ALS.

In addition to the $\Delta \mathrm{FS}$ score, age $\geq 70$ years, symptom duration $\leq 2$ years, ALSFRS-R total score $\leq 33$, ALSFRS-R respiratory subscore $<10$, and bulbar-onset disease were also established as potential adverse prognostic biomarkers in the current ALS cohort, albeit on univariable analysis. Interestingly, while there was a trend for riluzole therapy to increase survival in ALS, this was not significant in the current cohort and probably relates to sample size, with the majority of patients with ALS (77\%) receiving riluzole therapy. Importantly, multiple Cox proportional hazards modelling established that in addition to the $\Delta \mathrm{FS}$, site of disease onset was the only other independent prognostic biomarker of survival in ALS. While the site of disease onset could be utilised in patient care planning and stratification into clinical trials, the utility of site of disease onset as a biomarker of treatment efficacy appears limited when compared with the $\Delta \mathrm{FS}$ score, as $\Delta \mathrm{FS}$ is a numerical parameter that could be modulated by therapies, a notion that needs verification in future studies. It should be stressed, however, that the $\Delta \mathrm{FS}$ score would not necessarily determine the need for early intervention, such as insertion of PEG or the start of NIV, and that such decisions remain dependent on individual patient assessment and are likely to be determined by a number of factors.

A potential limitation of the present study is the absence of quantitative respiratory function data, including the forced vital capacity. Instead, we utilised the ALSFRS-R respiratory subscore as a measure of respiratory function at initial visit. While the presence of respiratory dysfunction adversely impacts on survival, ${ }^{18}$ previous studies did not establish a significant difference in the forced vital capacity between 'faster' and 'slower' progressing patients. ${ }^{11}$ Of further relevance, while the median ALSFRS-R respiratory subscore was significantly lower in patients with the fastest rate of disease progression $(\Delta \mathrm{FS}>1.11)$, it was found not to be an independent predictor of survival on multiple Cox proportional hazards analysis. Taken together, the present findings suggest that while patients with ALS with aggressive disease $(\Delta \mathrm{FS}>1.11)$ exhibit respiratory dysfunction at initial visit, significant respiratory dysfunction is unlikely, and typically manifests in later stages of the disease process.

It has also been argued that a prolonged presymptomatic period may be a feature of ALS, akin to that seen in other neurodegenerative disorders, with the pathogenic processes culminating in the ALS phenotype starting much earlier in life, perhaps at the time of conception. ${ }^{19}$ By contrast, others have suggested that the pathogenic processes develop just before or co-incidental with the clinical onset of ALS. ${ }^{20}{ }^{21}$ Irrespective of the length of the 'preclinical period', the findings in the present study suggest that once the disease starts, the $\Delta \mathrm{FS}$ score appears to be a useful prognostic biomarker.

In conclusion, the present study established the $\Delta \mathrm{FS}$ score as a sensitive and independent prognostic biomarker for ALS. Specifically, three prognostic $\Delta \mathrm{FS}$ subgroups emerged, all correlating with survival times. Importantly, the cut-off values for the prognostic subgroups were similar to the previously reported values which were arbitrarily derived from distinct ALS cohorts, supporting the utility of $\Delta \mathrm{FS}$ score as a ubiquitous prognostic biomarker in ALS. Future studies should assess whether the $\Delta \mathrm{FS}$ could be utilised as a biomarker of therapeutic effectiveness in ALS at early stages of drug development.

Funding Funding support from the Motor Neuron Disease Research Institute of Australia (MNDRIA) and Medical Research Council of Australia (Project grant number APP1055778) is gratefully acknowledged.

Competing interests None declared.

\section{Patient consent Obtained.}

Ethics approval This study was approved by the Sydney West Area Health Service and the St Vincent's Hospital Sydney Human Research Ethics Committees'.

Provenance and peer review Not commissioned; externally peer reviewed.

\section{REFERENCES}

1 Turner MR, Kiernan MC, Leigh PN, et al. Biomarkers in amyotrophic lateral sclerosis. Lancet Neurol 2009;8:94-109.

2 Kiernan MC, Vucic S, Cheah BC, et al. Amyotrophic lateral sclerosis. Lancet 2011;377:942-55.

3 Chio A. ISIS Survey: an international study on the diagnostic process and its implications in amyotrophic lateral sclerosis. J Neurol 1999;246(Suppl 3):1-5.

4 Mandrioli J, Faglioni P, Nichelli P, et al. Amyotrophic lateral sclerosis: prognostic indicators of survival. Amyotroph Lat Scler 2006;7:217-26.

5 Desport JC, Torny F, Lacoste M, et al. Hypermetabolism in ALS: correlations with clinical and paraclinical parameters. Neurodegener Dis 2005;2:202-7.

6 Lee JR-J, Annegers JF, Appel SH. Prognosis of amyotrophic lateral sclerosis and the effect of referral selection. J Neurol Sci 1995;132:207-15.

7 Cedarbaum JM, Stambler N, Malta E, et al. The ALSFRS-R: a revised ALS functional rating scale that incorporates assessments of respiratory function. BDNF ALS Study Group (Phase III). J Neurol Sci 1999;169:13-21.

8 Kollewe K, Mauss U, Krampfl K, et al. ALSFRS-R score and its ratio: a useful predictor for ALS-progression. J Neurol Sci 2008;275:69-73.

9 Gordon PH, Salachas F, Lacomblez L, et al. Predicting survival of patients with amyotrophic lateral sclerosis at presentation: a 15-year experience. Neurodegener Dis 2013;12:81-90.

10 Zoccolella S, Beghi E, Palagano G, et al. Analysis of survival and prognostic factors in amyotrophic lateral sclerosis: a population based study. J Neurol Neurosurg Psychiatry 2008;79:33-7

11 Kimura F, Fujimura $C$, Ishida $S$, et al. Progression rate of ALSFRS-R at time of diagnosis predicts survival time in ALS. Neurology 2006;66:265-7.

12 Gordon PH, Cheung YK. Progression rate of ALSFRS-R at time of diagnosis predicts survival time in ALS. Neurology 2006;67:1314-15.

13 Kiernan MC, Talman P, Henderson RD, et al. Establishment of an Australian motor neurone disease registry. Med J Aust 2006;184:367-8.

14 Brooks B, Miller R, Swash M, et al. El Escorial revisited: revised criteria for the diagnosis of amyotrophic lateral sclerosis. Amyotroph Lateral Scler Other Motor Neuron Disord 2000;1:293-9.

15 Traynor BJ, Alexander M, Corr B, et al. Effect of a multidisciplinary amyotrophic lateral sclerosis (ALS) clinic on ALS survival: a population based study, 1996-2000. J Neurol Neurosurg Psychiatry 2003;74:1258-61.

16 Vucic S, Rothstein JD, Kiernan MC. Advances in treating amyotrophic lateral sclerosis: insights from pathophysiological studies. Trends Neurosci 2014;37:433-42.

17 Nicholson K, Cudkowicz M, Berry J. Clinical trial designs in amyotrophic lateral sclerosis: does one design fit all? Neurotherapeutics 2015;12:376-83.

18 Czaplinski A, Yen AA, Appel SH. Forced vital capacity (FVC) as an indicator of survival and disease progression in an ALS clinic population. J Neurol Neurosurg Psychiatry 2006;77:390-2.

19 Eisen $A$, Kiernan $M$, Mitsumoto $H$, et al. Amyotrophic lateral sclerosis: a long preclinical period? J Neurol Neurosurg Psychiatry 2014;85:1232-8.

20 Aggarwal A, Nicholson G. Detection of preclinical motor neurone loss in SOD1 mutation carriers using motor unit number estimation. J Neurol Neurosurg Psychiatry 2002;73:199-201.

21 de Carvalho M, Swash M. The onset of amyotrophic lateral sclerosis. I Neurol Neurosurg Psychiatry 2006;77:388-9. 